\title{
ASSOCIATION OF CORTACTIN AND MDM2 IN SOLID AMELOBLASTOMA AND ITS MALIGNANT COUNTERPART
}

\author{
Nadia A. Radi*
}

\begin{abstract}
Ameloblastoma is characterized by heterogeneity regarding its pathogenetic development, molecular abnormalities, and clinical course. Determining the molecular basis underlying these important differences remains challenging. Nevertheless, identifying molecules that influence tumor behavior would be the first steps towards gaining some insights into its pathobiology. MDM2, a $90-95 \mathrm{kDa}$ protein coded by a mdm2 gene which is mapped on chromosome $12 \mathrm{q} 13-14$. MDM2 is a negative regulator of $\mathrm{p} 53$ activity, through binding to the transcriptional activation domain of $\mathrm{p} 53$ leading to proteasome-mediated degradation, Cortactin, a product of CTTN gene, mapped to human chromosome 11q13 regulates actin-related (Arp2/3) complex induction of actin polymerization during invadopodia formation, thereby promoting cell motility. This has been accomplished through its involvement in all steps of the invadosome lifecycle, from the assembly, maturation, proteolytic activity, and disassembly. This study aims to evaluate the association of both MDM2 and Cortactin expression in solid ameloblastoma and its malignant counterpart and analyzing their significance. 10 cases of $\mathrm{SAB}$ with its different histologic variants and 20 cases malignant variants of ameloblastoma tumors were collected. An immunohistochemical investigation using, Cortactin, and MDM2 antibodies were done for all specimens. There was a statistically significant difference between malignant group and benign group. According to the current study Cortactin and MDM2 can be useful when considering the diagnosis of malignancy.
\end{abstract}

Keywords: MDM2, Cortactin, Ameloblastoma, Malignant ameloblastoma

\section{INTRODUCTION}

Odontogenic tumors are a varied group of lesions with different clinical behavior and histopathologic types, ranging from hamartomatous lesions to malignancy ${ }^{(1)}$. Among the pathological entities that affect the maxillofacial region, ameloblastoma is the second most common odontogenic tumor ${ }^{(2,3)}$. Although considered a benign neoplasm, it deserves special attention because of its particular biological behavior exhibiting greater infiltrative potential, a highly destructive tumor of the jaw, high recurrence rate and capacity to metastasize or undergo malignant transformation ${ }^{(4)}$. Ameloblastoma is characterized by heterogeneity regarding its pathogenetic development, molecular abnormalities, and clinical course. Determining the molecular basis underlying

* Lecturer of Oral and Dental Pathology, Faculty of Dental Medicine for Girls. Al Azhar University. 
these important differences remains challenging. Nevertheless, identifying molecules that influence tumor behavior would be the first steps towards gaining some insights into its pathobiology ${ }^{(5,6)}$. Malignant ameloblastoma is a histologically benignappearing ameloblastoma that has metastasized. Metastases are usually localized in the regional lymph nodes or distant organs most commonly the lungs and retain the benign characteristics of the primary neoplasm. Thus, whether metastasis will occur or not cannot be predicted based on histology. While ameloblastic carcinoma is a highly aggressive malignant tumor displaying cellular atypia even if metastases are absent ${ }^{(7,8)}$.

Normal cell growth is regulated by a finely tuned balance between its growth promoting proto-oncogene and its growth constraining gene. Disturbance in this equilibrium either from an activated oncogene or an inactivated growth suppressing gene may propel the cells toward aggressiveness. Determination of this epithelial proliferation helps in investigating the differences between biological behaviors of various tumors. One such specific marker to identify proliferative activity and tumor aggressiveness is MDM2, a 90$95 \mathrm{kDa}$ protein coded by a mdm 2 gene which is mapped on chromosome 12q13-14. MDM2 is a negative regulator of p53 activity, through binding to the transcriptional activation domain of p53 leading to proteasome-mediated degradation, so the overproduction of MDM2 protein gives rise to a greater risk in the development of malignancy. Increased expression of MDM2 has been reported in several human cancers including breast carcinomas, malignant melanomas, esophageal carcinomas, and head and neck cancers and associated with poor prognosis ${ }^{(4,9,10)}$.

Cell migration is essential in many biological processes as well as in disease progressions such as cancer invasion and metastasis. It is a multistep mechanism initiated by the formation of cellular membranous protrusions. These protrusive structures are termed filopodia, lamellipodia, invadopodia and podosomes based on their morphological, structural and functional properties. Invadopodia are finger-like ventral membranous protrusions formed by invasive tumor cells during migration ${ }^{(11-14)}$. Cortactin, a product of CTTN gene, mapped to human chromosome 11q13 regulates actin-related (Arp2/3) complex induction of actin polymerization during invadopodia formation, thereby promoting cell motility. This has been accomplished through its involvement in all steps of the invadosome lifecycle, from the assembly, maturation, proteolytic activity, and disassembly. Cortactin deregulation is associated with increased invasion and metastases of breast, oesophageal, renal, head and neck cancers. Its overexpression has been correlated with advanced clinic pathological stage and poor patient prognosis and decreased survival ${ }^{(15-24)}$. Therefore, this study aims to evaluate the association of both MDM2 and Cortactin expression in solid ameloblastoma and its malignant counterpart and analyzing their significance.

\section{MATERIALS AND METHODS}

\section{Case selection}

The specimens for this study were retrieved as paraffin-embedded blocks from the archives of Oral Pathology Department, Faculty of Dentistry, Alexandria University. The specimens were divided into two groups according to WHO classification. Group I, (10 cases of SAB with its different histologic variants) and group II, 20 cases malignant variants of ameloblastoma tumors (10 cases of malignant ameloblastoma and 10 cases of ameloblastic carcinoma).

\section{Histological analysis}

Using H\&E for reevaluation of the aforementioned cases was carried out to confirm their diagnosis, and reclassifying them according to $\mathrm{WHO}^{(25)}$. 


\section{Immunohistochemical analysis}

Immunohistochemical staining was performed on $4-\mu \mathrm{m}$ thick formalin-fixed paraffin-embedded sections. Sections were deparaffinized in xylene and then rehydrated in graded ethanol solutions. To block endogenous peroxidase activity, sections were treated with 3\% hydrogen peroxide solution for 20 minutes. Antigen retrieval was accomplished by microwaving at $99^{\circ} \mathrm{C}$ in $10 \mathrm{mM}$, citrate buffer (pH 6.0) for $20 \mathrm{~min}$. Consequently, the sections were incubated with primary monoclonal antibodies according to the manufacturer's instructions using Cortactin, and MDM2 mouse monoclonal antibodies [(Clone A-4 Cat.No. SC-55578; and Clone 1B10). Respectively and were manufactured by Dako, Denmark. The dilution used was 1:50 for both markers in phosphate buffered saline. (26) Detection was carried out using the universal kit (DAKO, Denmark) by washing slides in PBS for 5 minutes and incubated with a secondary antibody that was biotinylated goat serum conjugated rabbit and mouse sera for 30 minutes. Sections were then washed for 5 minutes in PBS followed by the development of antigen-antibody visualization by diaminobenzidine [DAB] in PBS containing $40 \% \mathrm{H}_{2} \mathrm{O}_{2}$. Sections were washed under running tap water for 10 minutes, then counterstained with Mayer's hematoxylin and mounted. All the steps were carried out at room temperature and after each step, the sections were washed with PBS, pH 7.2.

\section{Histomorphometric analysis}

Immunoreactivity, for MDM2 and Cortactin; were evaluated by estimating the area percentage of positive immunostained cells in relation to the area examined in each field using Leica image analyzer computer system (Germany) at Oral and Dental Pathology Department, Faculty of Dental Medicine for Girls, Al Azhar University, Cairo. The area percentage of antibodies' reactive areas were measured with reference to a standard measuring frame of area $11434.9 \mu \mathrm{m} 2$ using a magnification (x 200). Using the color detection, reactive areas of positive immunostaining were masked by a blue binary color. Ten fields per each slide section were successively taken, to be histomorphometrically evaluated. Mean values were then obtained for each specimen.

\section{Statistical analysis}

Data were represented as means, median, standard deviation (SD), minimum and maximum values; Analysis of variance (ANOVA) test was used for statistical analysis of the difference between groups. Tukey's post hoc test was performed as an ANOVA test revealed a significant difference. The unpaired test was used for comparison between the two malignant variants. Mann-Whitney $U$ test was used to compare between benign and malignant groups. The significance level was set at $p \leq 0.05$. Statistical analysis was performed with SPSS 16.0 (Statistical Package for Scientific Studies, SPSS, Inc., Chicago, IL, USA) for Windows. Spearman's correlation coefficient was used to determine correlations between different markers.

\section{RESULTS}

\section{Histopathological findings}

SAB has five different histopathological variants. They are follicular ameloblastoma presented as multiple islands of odontogenic epithelial cells. The peripheral cells are tall columnar with reverse polarity and central stellate reticula like cells surrounded by connective tissue stroma, while in an acanthomatous variant the neoplastic island showed central stellate reticula like cells with formation of squamous cells having eosinophilic cytoplasm and keratin formation. Basal cell ameloblastoma composed of islands of uniform compacted dark stained cuboidal basaloid cells surrounded by juxtaepithelial hyalinized homogenous fibrous tissue band. Granular cell ameloblastoma showed tumor islands with peripheral columnar cells surrounding central large cells with granular 
eosinophilic cytoplasm and peripheral nuclei. Plexiform ameloblastoma showed anastomosing cords of ameloblastoma epithelium, the peripheral cells are cuboidal and the central cells are few loosely arranged stellate reticulum like cells within degenerated connective tissue stroma.

Malignant ameloblastoma showed differentiated ameloblastoma without obvious cytological atypia. Ameloblastic carcinoma showed areas exhibit squamous differentiation with obvious cytological atypia as cellular pleomorphism, nuclear hyperchromatism, and abnormal mitosis. The connective tissue stroma showing increased vascularity with engorged blood vessels some of these blood vessels were invaded by detached malignant cells (Fig., 1, A-G)

\section{Immunohistochemical findings:}

Cortactin immunostaining was seen in the cytoplasm and/or cell membrane of the peripheral basal and stellate-reticulum like cells of the neoplastic islands of all histopathological variants of solid ameloblastoma while it was negative in central squamous cells of acanthomatous type. Few connective tissue fibroblasts showed positive immunostaining. While in malignant ameloblastoma and ameloblastic carcinoma cortactin immunopositivity was seen in the cytoplasm and/or cell membrane of neoplastic cells. MDM2 immunostaining was seen in the nucleus and cytoplasm of peripheral cells and central stellate reticulum like cells of neoplastic islands of all histopathological variants of solid ameloblastoma while it was negative in the central squamous cells of acanthomatous type. Some connective tissue fibroblasts showed positive nuclear immunostaining. In malignant ameloblastoma and ameloblastic carcinoma, MDM2 immunostaining was seen on the nucleus and cytoplasm of neoplastic cells.[Fig., 2, (H-N, Cortactin immunostaining. O-U, MDM2 immunostaining)].

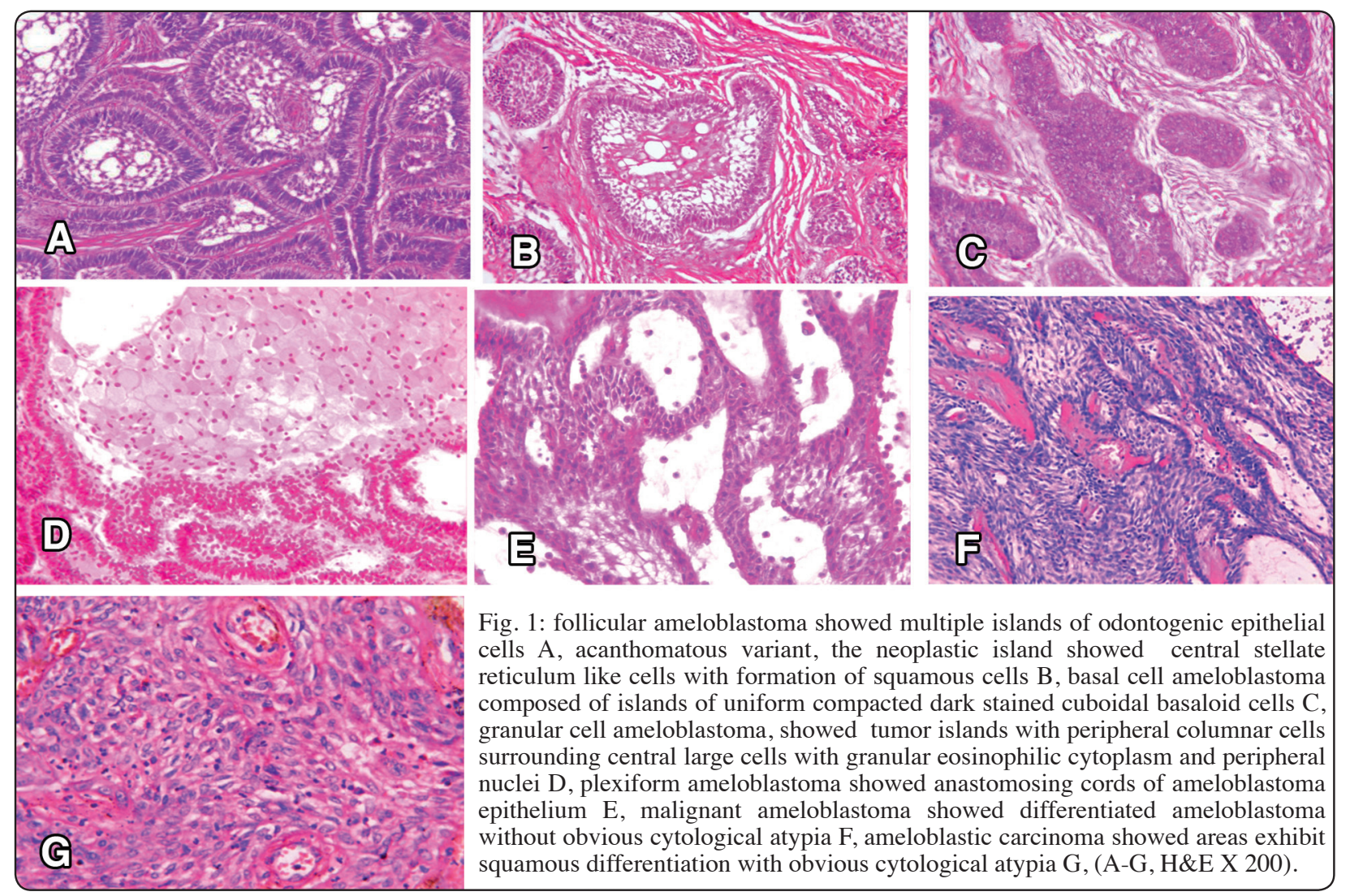




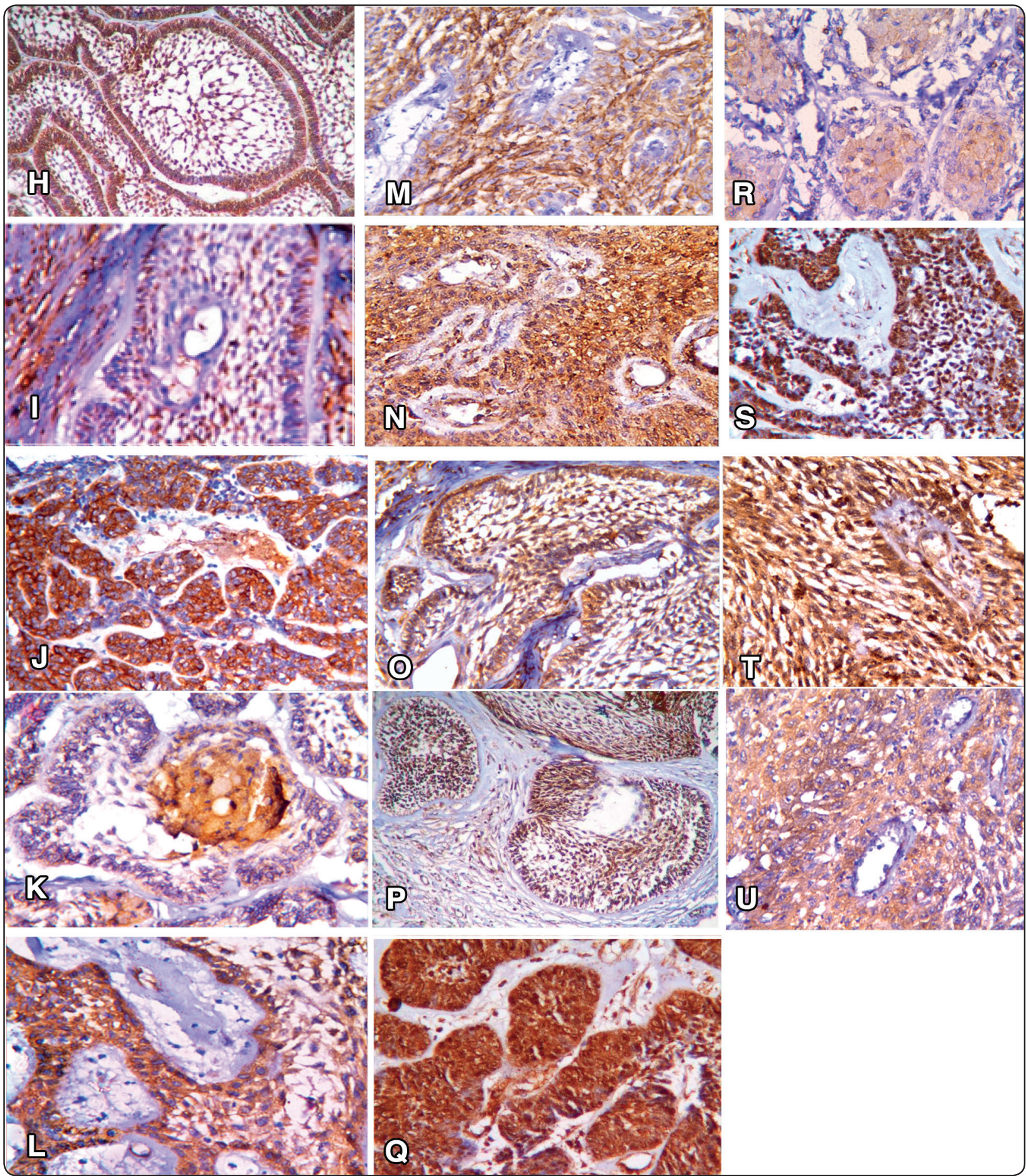

Figure 2:, Cortactin immunostaining was seen in the cytoplasm and/or cell membrane of the peripheral basal and stellate-reticulum like cells of the neoplastic islands of all histopathological variants of solid ameloblastoma while it was negative in central squamous cells of acanthomatous type. In malignant ameloblastoma and ameloblastic carcinoma the immunopositivity was seen in cytoplasm and/or cell membrane of neoplastic cells (H-N, Cortactin immunostaining, X 200). MDM2 immunostaining was seen in the nucleus and cytoplasm of peripheral cells and central stellate reticulum like cells of neoplastic islands of all histopathological variants of solid ameloblastoma while it was negative in the central squamous cells of acanthomatous type. In malignant ameloblastoma and ameloblastic carcinoma MDM2 immunostaining was seen on the nucleus and cytoplasm of neoplastic cells (O-U, MDM2 immunostaining, X 200). 


\section{Statistical Results}

Statistically, for both markers there was no statistically significant difference between malignant ameloblastoma and ameloblastic carcinoma; both showed the statistically significantly highest mean area percent. But there was a statistically significant difference between malignant group and benign group (Table 1,2. Fig., 3,4). Pearson correlation test revealed a statistically significant direct correlation between Cortactin and MDM2(Table 3, Fig., 5).

TABLE (1): Comparison between cortactin area \% in benign and malignant groups by Mann-Whitney U test.

\begin{tabular}{ccccccc}
\hline Group & Mean & SD & Median & Minimum & Maximum & $P$-value \\
\hline Benign & 31.60 & 9.14 & 33.92 & 14.74 & 44.13 & $<0.001^{*}$ \\
Malignant & 62.69 & 4.56 & 61.12 & 56.84 & 69.55 & \\
\hline
\end{tabular}

\section{*: Significant at $P \leq 0.05$}

TABLE (2): Comparison between MDM2 area \% in benign and malignant groups by Mann-Whitney U test.

\begin{tabular}{ccccccc}
\hline Group & Mean & SD & Median & Minimum & Maximum & $P$-value \\
\hline Benign & 34.62 & 8.84 & 38.29 & 18.98 & 45.76 & $<0.001^{*}$ \\
Malignant & 65.13 & 2.65 & 64.98 & 60.81 & 68.67 & \\
\hline
\end{tabular}

\section{*: Significant at $P \leq 0.05$}

TABLE (3): Correlation between Cortactin and MDM2 by Pearson correlation test

\begin{tabular}{lll}
\hline Markers & Correlation coefficient & $P$-value \\
Cortactin and MDM2 & 0.888 & $<0.001 *$ \\
\hline
\end{tabular}

\section{*: Significant at $P \leq 0.05$}

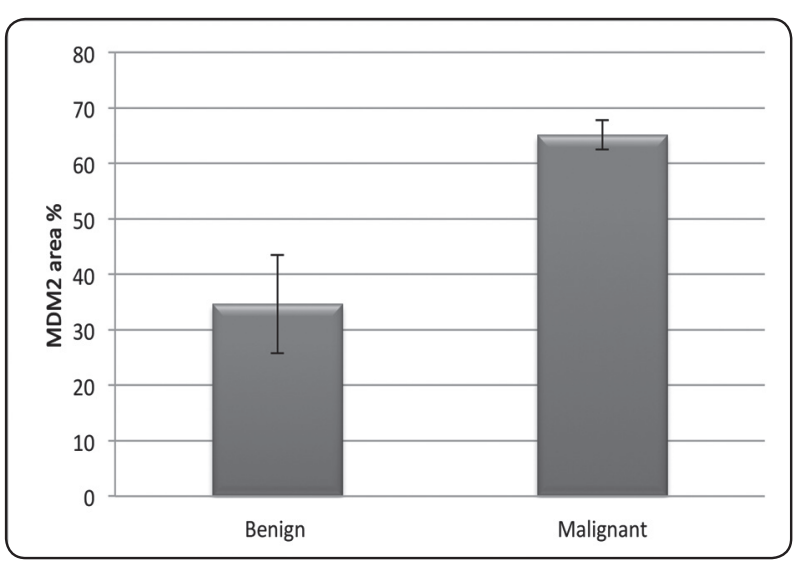

Fig. (3) Bar chart representing mean values for cortactin area \% in benign and malignant groups.

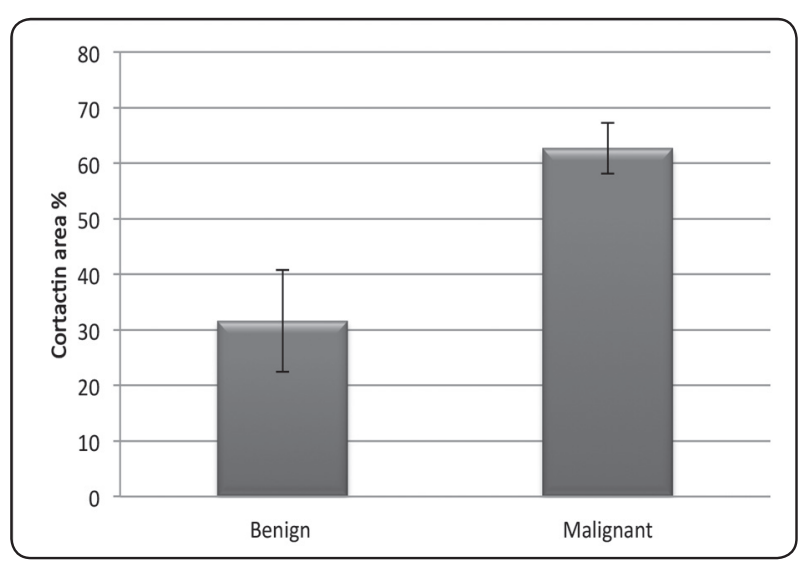

Fig. (4) Bar chart representing mean values for MDM2 area \% in benign and malignant groups. 


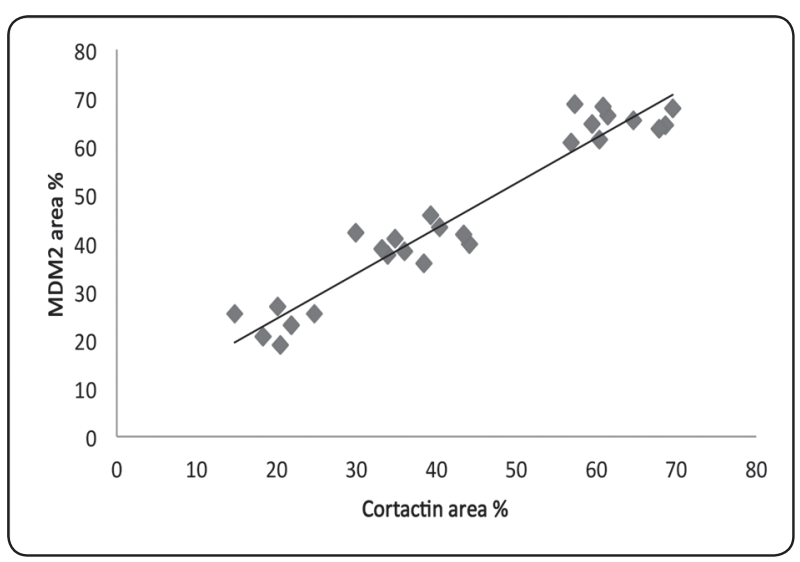

Fig., (5) Scatter diagram showing a correlation between cortactin and MDM2.

\section{DISCUSSION}

The rarity of malignant odontogenic tumors, variations in their pathogenesis and biological behavior all contribute to the difficulty in diagnosing them. Furthermore, it may be difficult to differentiate some of these tumors from their benign counterparts and occasional difficulty in confirming their odontogenic origin may also contribute to the challenge of diagnosing these malignant tumors. The most obvious characteristics of malignant tumors are proliferation and migration. So, the present study was designed to evaluate the immunohistochemical expression of cortactin, and MDM2 in solid ameloblastoma and malignant counterpart, correlating their expression to each other and also, predict their prognostic value in these tumors.

Concerning SAB, cortactin immunostaining was seen in the peripheral and central stellate cells of the tumoral epithelial islands while it was negative for squamous area and keratin in the acanthomatous subtype. These results were in accordance with other studies that showed acanthomatous epithelial tumors were negative to invadopodia proteins and they explained this as tumor areas with strong invadopodia protein expression denotes sites of dynamic actin remodeling whereas negative areas signify a shift to quiescence. ${ }^{(27,28)}$ Also, cortactin immunolocalization was cytoplasmic and membranous. This finding was explained as cortactin concentrates at the cell membrane to initiate invadopodia assembly. In addition, cortactin can bind to actin-related molecules complexes that may possibly function as bridges in the initial steps of invadopodia formation. It was revealed that cell migration and extracellular matrix degradation influence ameloblastoma behavior as the local invasiveness of ameloblastoma is dependent upon the migratory potential of its tumor cells as defined by their distribution of cortactin, other invadopodia proteins and membrane-type 1 MMP (MT-MMP) recruitment. ${ }^{(3,29,30)}$

Regarding ameloblastic carcinoma and malignant ameloblastoma, the mean value of cortactin immunoexpression of ameloblastic carcinoma was higher than that of malignant ameloblastoma with no significant difference between them. These results were in accordance with another study where cortactin immunostaining was higher in poorly differentiated non-small cell lung carcinoma when compared with a well-differentiated counterpart with no significant difference. Also, cortactin positive immunostaining was seen in a blood vessel in malignant variants of $A B$. These results were in agreement with the previous study where expression of cortactin was found in endothelial cells of blood vessels of colorectal carcinoma. An important mechanism that regulates endothelial junctions and thus vascular permeability is the remodeling of the actin cytoskeleton so cortactin is essential for the proper control of endothelial barrier integrity, permeability and maintaining endothelial monolayer integrity ${ }^{(31,32)}$.

In the present study, the distributions of cortactin positive immune reaction were variable among the studied tumors, where malignant variants had higher mean values with highly significant difference than benign tumors. These results were in accordance with the previous study where the 
immunopositivity for cortactin was significantly higher in epithelial ovarian cancer compared with benign ovarian tumor. High expression of cortactin was related to poorer differentiation, greater tumor cell motility, and greater malignancy. As cortactin improve the capability of the cells to produce dominant invadopodia in response to stimulation of growth factor which promote the cellular motility and ability to migrate. Also, cortactin recruits ECM-degrading proteinases MMP to invadopodia sites, producing enhanced ECM degradation and invasiveness ${ }^{(33,34)}$.

Regarding SAB, the positivity for MDM2 was observed in nucleus and cytoplasm of the peripheral and central stellate reticulum like cells. These results were in agreement with a previous study representing the expression of MDM2 was almost confined to nuclei except cytoplasmic staining was observed sometimes. The MDM2 positive nuclei were found predominantly in peripheral ameloblastlike cells and it was also seen in central stellate reticulum like cells suggesting that the proliferative activity of ameloblastomas is essentially restricted to the peripheral cells $\mathrm{s}^{(4)}$.

Concerning MDM2 results in the current study, there was no significant difference between malignant ameloblastoma and ameloblastic carcinoma, but malignant variants had higher significant difference than benign tumors where ameloblastic carcinoma showed the statistically significant highest mean value. These results were in accordance with a former study that showed MDM2 positive expression in SCC was significantly higher than in normal oral mucosa and oral leukoplakia. Also, MDM2 expression in the lymph node metastasis was significantly higher than that without lymph node metastasis. This explained by the fact of MDM2 mainly exerts its activity by downregulating the P53 tumor suppressor gene activity and its product. So high expression levels of MDM2 decrease p53 protein levels and function, leading to accelerated tumor formation and progression ${ }^{(35)}$.
In the present study, malignant ameloblastoma and ameloblastic carcinoma showed positive cytoplasmic and nuclear immunostaining of MDM2. These results were in accordance with another study where ameloblastic carcinomas were diffusely positive nuclear and cytoplasmic for MDM2. The association of both cytoplasmic and nuclear immunostaining exhibited more aggressive features than nuclear immunostaining alone ${ }^{(36,37)}$.

The most obvious characteristics of malignant tumors are proliferation and migration. So there is a statistically significant positive correlation between cortactin and MDM2 with respect to a malignant variant of ameloblastoma as both markers increase in malignant compared to benign tumors.

\section{CONCLUSION}

Cortactin and MDM2 are useful when considering the diagnosis of malignancy in odontogenic tumors

\section{REFERENCES}

1. Wright JM, Soluk Tekkesin M. Odontogenic Tumors. Where are we in 2017?. J Istanb Univ Fac Dent. 2017;51: S10-S30

2. Adriane S, Siqueira AS, Carvalho MRD. Matrix metalloproteinases, TIMPs and growth factors regulating ameloblastoma behavior. Histopathology 2010; 57; 128-137.

3. Pinheiro JJV, Nascimento CF, Freitas VM, de Siqueira AS, Junior SM, Jaeger RG. Invadopodia proteins, cortactin and membrane type I matrix metalloproteinase (MT1-MMP) are expressed in ameloblastoma. Histopathology. 2011; 59: 1261-79.

4. Krishna A, Kaveri H, Naveen Kumar RK, Kumaraswamy KL, Shylaja S, Murthy S. Overexpression of MDM2 protein in ameloblastomas as compared to the adenomatoid odontogenic tumor. J Cancer Res Ther. 2012; 2: 232-7.

5. Jhamb J, Kramer J. Molecular concepts in the pathogenesis of ameloblastoma: implications for therapeutics. Exp Mol Pathol. 2014; 97: 345-53.

6. Chong HS, Zainal AR, Hidetsugu T, Kamila MA, Hitoshi N, Kok HN: Invadopodia proteins, cortactin, N-WASP, and WIP differentially promote local invasiveness in ameloblastoma J Oral Pathol Med (2016) 45: 591-598. 
7. Kodati S,Majumdar S, Uppala D, Namana M.Ameloblastic carcinoma: A report of three cases. J Clin Diagn Res. 2016;10: 23-25.

8. Valkadinov I, Conev N, Dzhenkov D, Donev I. Rare case of ameloblastoma with pulmonary metastases. Intractable Rare Dis Res. 2017; 6: 211-4.

9. Rayburn E, Zhang R, He J, Wang H. MDM2, and human malignancies: Expression, clinical pathology, prognostic markers and implications for chemotherapy. Curr Cancer Drug Targets 2005; 5: 27-41.

10. Bose I, Ghosh B. The p53-MDM2 network: From oscillations to apoptosis. J Biosci. 2007; 32: 991-7.

11. Yamaguchi H, Condeelis J. Regulation of the actin cytoskeleton in cancer cell migration and invasion. Biochim Biophys Acta 2007; 1773: 642-52.

12. Stevenson RP, Veltman D, Machesky LM. Actin-bundling proteins in cancer progression at a glance. J Cell Sci 2012; 125: 1073-9.

13. Beaty BT, Condeelis J. Digging a little deeper: the stages of invadopodium formation and maturation. Eur $\mathrm{J}$ Cell Biol 2014; 93: 438-44.

14. Murphy DA, Courtneidge SA. The 'ins' and 'outs' of podosomes and invadopodia: characteristics, formation, and function. Nat Rev Mol Cell Biol 2012; 12: 413-26.

15. Buday L, Downward J. Roles of cortactin in tumor pathogenesis. Biochim Biophys Acta 2007; 1775: 263-73.

16. Shen HL, Liu QJ, Yang PQ. Protein interactions of cortactin in relation to invadopodia formation in metastatic renal clear cell carcinoma. Tumor Biol. 2015; 36: 3417-22.

17. Hsu NY, Yeh KT, Chiang IP. Cortactin overexpression in the esophageal squamous cell carcinoma and its involvement in the carcinogenesis. Dis Esophagus 2008; 21: 402-8.

18. Clark ES, Brown B, Whigham AS, Kochaishvili A, Yarbrough WG, Weaver AM. Aggressiveness of HNSCC tumors depends on expression levels of cortactin, a gene in the 11q13 amplicon. Oncogene. 2009; 28: 431-44.

19. Ambrosio EP, Rosa FE, Domingues MAC. Cortactin is associated with perineural invasion in the deep invasive front area of laryngeal carcinomas. Hum Pathol. 2011; 42: 1221-9.

20. Yamada S, Yanamoto S, Kawasaki G, Mizuno A, Nemoto TK. Overexpression of cortactin increases invasion potential in oral squamous cell carcinoma. Pathol Oncol Res. 2010; 16: 523-31.

21. DesMarais V, Yamaguchi H, Oser M. N-WASP, and cortactin are involved in invadopodium-dependent chemotaxis to EGF in breast tumor cells. Cell Motil Cytoskeleton. 2009; 66: 303-16.

22. Martini V, Gattazzo C, Frezzato F, Trimarco V, Pizzi M, Chiodin G, Severin F, Piazza F, Brunati AM, Semenzato G, Trentin L. Cortactin, a Lyn substrate, is a checkpoint molecule at the intersection of BCR and CXCR4 signaling pathway in chronic lymphocytic leukaemia cells. Br J Haematol. 2017; 178: 81-93.

23. MacGrath SM, Koleske AJ. Cortactin in cell migration and cancer at a glance. J Cell Sci. 2012; 125: 1621-6.

24. Radhakrishnan VM, Kojs P, Young G, Ramalingam R, Jagadish B, Mash EA, Martinez JD, Ghishan FK, Kiela PR. pTyr ${ }^{421}$ Cortactin is overexpressed in colon cancer and is dephosphorylated by curcumin: Involvement of nonreceptor type 1 protein tyrosine phosphatase (PTPN1). PLoS One. 2014; 22: 57-96.

25. El-Naggar AK, Chan JK, Grandis JR, Takata T, Slootweg PJ. WHO Classification of Head and Neck Tumours. 4th ed. Lyon, France: IARC Press; 2017

26. Ramos-Vara JA, Miller MA. Technical aspects of immunohistochemistry. Vet Pathol. 2014; 51:42-87.

27. Stevenson RP, Veltman D, Machesky LM. Actin-binding proteins in cancer progression at a glance. J Cell Sci. 2012;125: 1073-9.

28. Beaty BT, Condeelis J. Digging a little deeper: the stages of invadopodium formation and maturation. Eur J Cell Biol. 2014; 93: 438-44.

29. Ribeiro Ribeiro AL, da Costa NM, de Siqueira AS, Brasil da Silva W,. Keratocystic odontogenic tumor overexpresses invadopodia-related proteins, suggesting invadopodia formation. Oral Surg Oral Med Oral Pathol Oral Radiol. 2016;122: 500-8.

30. Siar CH, Rahman ZA, Tsujigiwa H, Mohamed Om Alblazi K, Nagatsuka H, Ng KH. Invadopodia proteins, cortactin, N-WASP and WIP differentially promote local invasiveness in ameloblastoma. J Oral Pathol Med. 2016; 11: $12-41$.

31. Fawzy AI, Gayyed MF, Tawfik NR. Cortactin and EGFR Proteins expression in Egyptian patients with non-small cell lung carcinoma. Int J of Cancer Res. 2013; 9: 24-35. 
32. Kim YN, Choi JE, Bae JS, Jang KY, Chung MJ, Moon WS, Kang MJ, Lee DG, Park HS. Expression of cortactin and focal adhesion kinase in colorectal adenocarcinoma: correlation with clinicopathologic parameters and their prognostic implication. Korean J Pathol. 2012; 46: 454-62.

33. Li A, Zhang L, Zhang X, Jin W, Ren Y. Expression and clinical significance of cortactin protein in ovarian neoplasms.Clin Transl Oncol. 2016;18: 220-7.

34. Yin M, Ma W, An L. Cortactin in cancer cell migration and invasion. Oncotarget. 2017; 8: 232-43.
35. Cui JJ, Han XL, Wang WM. Expression and significance of p53 and $\mathrm{mdm} 2$ in patients with leukoplakia cancer. Asian Pac J Trop Med. 2013; 6: 831-4.

36. Park HS, Park JM, Park S, Cho J, Kim SI, Park BW. Subcellular localization of Mdm2 expression and prognosis of breast cancer. Int J Clin Oncol. 2014; 19: 842-51.

37. Urso L, Calabrese F, Favaretto A, Conte P, Pasello G. Critical review about MDM2 in cancer: Possible role in malignant mesothelioma and implications for treatment. Crit Rev Oncol Hematol. 2016; 97: 220-30. 